left eyebrow. The edges of the wound on the arm came together without difficulty and the satisfactory manner in which the grafts have taken on the elbow is well shown in Fig. 4.

Dr. D. J. Morgan, pathologist to the hospital, examined the patehes under the microscope and found them to be typical of lupus.

Though sufficient time has not elapsed to warrant the statement that recovery will be permanent, yet so far everything points in this direction, no signs of recurrence being seen seven months after the operation. About two or three months after the operation the scar tissue on the arm and at the junction of the grafts over the elbow assumed a keloid condition, which can be seen in Fig. 4, but this seems to be now subsiding and is of no consequence. This history, I think, shows conclusively that cases of lupus which are not too far advanced for operation can be satisfactorily treated by excision, for which the following advantages may be claimed over treatment by the Finsen light or $x$ rays : (1) it is more radical ; (2) it saves time ; and (3) it is less expensive. In conclusion, I tender my thanks to Dr. J. T. English for kindly taking the photographs from which the accompanying illustrations have been reproduced.

Wimpole-street, W.

\section{A CASE OF CONGENITAL HEART LESION.}

BY WALTER BROADBENT, M.D. CANTAB., M. R O.P. LOND.,

ASSISTANT PHYSICTAN, SUSSEX COUNTY HOSPITAL.

THE following case is interesting as illustrating various points in the clinical history of congenital malformation of the heart. It has some bearing also on the question of the pulmonic systolic murmur; notbing is more common than a systolic murmur over the pulmonary artery, while actual stenosis of the orifice is extremely rare.

The patient, a girl, aged 13 years, was brought by her mother for feverishness and diarrhoea. The child was well nourished but was of a deep blue colour about the cheeks and the lips; this the mother had noticed for the last seven years and had been told that it was due to congenital heart mischief. There were also patches of deep freckle-coloured staining on the cheeks. The ends of the fingers were clubbed. A chain of much enlarged glands ran down each side of the neck. The pulse was 80 and not quite regular ; the artery was of normal size and easily compressible. Slight pulsation was visible in the second and third spaces, just to the left of the sternum. There was cardiac dulness in the second space for three-quarters of an inch from the sternum and down to the fifth space, where it extended from three quarters of an inch to the right of the sternum to half an inch outside the left vertical nipple line. At the apex and over the tricuspid area a first sound and loud second sound were heard and over the pulmonic area a first sound, high-pitched, blowing systolic murmur, and a much accentuated and reduplicated second sound. The systolic murmur was heard down the sternum to the fourth rib and for two inches to the left, and was not appreciably affected by her taking a deep breath. At the aortic cartilage a first sound and loud second sourd were heard. Movement of the left front was not qu te so good as of the right and resonance at the left apex was rather higher pitched. The air entry was diminished but there were no crepitations. She was very short of breath on the slightest exertion. The abdomen was distended and tender, especially below the umbilicus. A large gland could be felt in the left iliac region. There was also shifting dulness in the flanks and for an inch above the pubes but no fluid thrill was obtained. The temperature was $99^{\circ} \mathrm{F}$. but rose to $1005^{\circ}$ in the evening.

When in bed the girl's face lost its blue colour and was a deep red. Her temperature varied between $98^{\circ}$ and $101^{\circ}$. She was kept in bed and treated for the peritonitis. After six weeks the temperature was rarely above $99^{\circ}$. The abdomen was no longer distended or tender and the fluid had disappeared. The enlarged glands in the neck and abdomen were smaller. Also the systolic murmur in the pulmonic area was distinctly less loud. Two weeks later when allowed up for an hour a day she did not become blue and a month later still she was up all day and able to take more exercise than ever before without becoming at all blue, though she was soon short of breath on running. The pulse was regular, the systolic murmur was still present but much less loud and almost obliterated by deep inspiration, and the pulmonic second sound was only slightly accentuated, but still reduplicated.

The diagnosis of the heart lesion presents points of interest owing to the unexpected non-appearance of the blueness. A systolic murmur, which may be exceedingly loud, at and to the left of the pulmonic region accompanied by an accentuated second sound is often due to the lung not covering the conus arteriosus and the pulmonary artery, which will then lie against the under surface of the ribs. Vibrations are thus produced which are readily conducted by the chest wall. This murmur often varies with position and is usually considerably modified by the patient taking a deep breath and bringing a cushion of lung between the artery and the chest wall ; it may indeed completely disappear even when very loud. A systolic murmur accompanied by a loud second sound, whether aortic or pulmonic, is obviously not due to stenosis, and there can have been in this case no constriction at the pulmonic orifice, since the second sound would have been obliterated and not accentuated. The systolic murmur might have been caused in this child by patency of the ductus arteriosus and the accentuated and reduplicated second sound by a partial blocking of the pulmonary blood stream when the aortic blood entered the pulmonary artery, but a patent ductus arteriosus could not alone have produced the blueness, though its obliteration during the quiet of the long rest in bed would have explained the practical disappearance of the murmur. Or narrowing of the pulmonary artery at the point of entrance of the ductus arteriosus might have caused the systolic murmur with accentuated second sound and the blueness from deficient aeration of the blood, but rest in bed could not have had any curative effect and such a narrowing is exceedingly rare.

Patency of the foramen ovale causes no murmur, but this or pulmonic valvular stenosis is present in apparently all congenital cardiac defects accompanied by cyanosis. Patent foramen ovale may cause no symptoms at all until some disease of the lungs, such as bronchitis, raises the pressure on the right side of the heart and then cyanosis occurs unexpectedly early because blood from the right auricle flows into the left. 'This seems to explain the above case. When first seen the child had a dilated right ventricle, as evidenced by the cardiac dulness to the right of the lower sternum. Either old damage to the upper lobe of the left lung or enlarged mediastinal glands had caused obstruction to the pulmonary circulation. The prolonged rest in bed enabled the right ventricle to recover and to take the pressure off the right auricle, so that blood ceased to pass into the left auricle and perhaps the foramen ovale became smaller. The murmur may have been due to the want of expansion of the left lung uncovering the pulmonary artery or possibly there was some patency of the ductus arteriosus as well as of the foramen ovale.

Brighton.

\section{Climial allotes:}

MEDICAL, SURGICAL, OBSTETRICAL, AND THERAPEUTICAL.

\section{A CASE OF ACUTE FULMINATING GLAUCOMA} ENDING IN TOTAL BLINDNESS IN 18 HOURS AFTER ONSET AND OCOURRING DURING THE COURSE OF SEVERE SYPHILIS.

By the late ARThur T. White, M R.C.S. ENG., LATE PBINCIPAI MEDICAL OFFICER TO THE NILE RESERVOIR HOSPITAL, ASSOUAN, UPPER EGYPT.

THE following case of acute glaucoma ending in absolute blindness within 18 hours of onset is interesting, partly from the fact that fulminating glaucoma is not a common condition; partly from the absence, apart from secondary syphilis of a severe nature, of a cause; and partly from the circumstances that rendered the treatment difficult. At the 
moment, having no access to a reference library, I speak with diffidence, but so far as I have read and have been able to discover from the books at hand syphilis is not mentioned as a direct cause of glaucoma except by way of iritis.

The patient, an Italian labourer, aged 36 years, was admitted into the Nile Reservoir Hospital at Assouan on Feb. 2nd, 1901. I had been told that a man was lying ill at the works in one of Messrs. Aird's barracks and that he was1 vomiting and in great pain. When I saw him in the room he was obviously suffering from acute purulent conjunctivitis of both eyes; he was rolling about with pain in his supra-orbital and right temporal regions, and from time to time he vomited spasmodically a watery bile-stained fluid. I was told by his comrades that he began to vomit and to complain of the severity of the pain about 18 hours before I was called to him, though "for some time" he had been ailing and not at work, partly from his "bad eyes" and partly from syphilis from which he was then suffering.

On examination at the hospital the patient was found to be literally covered with secondary macular and papular syphilides. He was very anæmic and looked ill and thin and it was elicited that he had been living for some time past on the charity of other Italian workmen and had been getting very little to eat. He complained of intense pain over the brow and in the right temple and on more careful examination he was found to have the symptoms of acute glaucoma of the right eye which was of stony hardness with an anæsthetic and ground glass cornea, injected episcleral vessels, and absolutely no perception of light. It was very difficult to examine the eye owing to the severe purulent conjunctivitis with its accompanying chemosis. The tension in the left eye was normal. According to his own account his vision had been good, apart from a certain amount of sub. acute conjunctivitis, till about 24 hours before when he began to get a fogged vision which was rapidly followed by the severe browache and spasmodic vomiting and a rapid increase in the conjunctivitis. Owing to the purulent inflammation of his conjunctiva any operative measure such as iridectomy or sclerotomy seemed to me to be impossible and I had to be content with the administration of morphia hypodermically, cocaine and eserine instillation, and hot applications locally, with at the same time active anti-syphilitic treatment by free mercurial inunction, a generous diet, and attention to his general miserable condition. There was no remission or improvement in vision, though the eye gradually became less painful and somewhat less tense and the cornea cleared slightly: when he left the hospital the affected eye was stone blind. Beyond the conjunctivitis nothing could be detected in the remaining sound eye as regards its tension, its visual acuity, or the condition of the fundus.

The previous history of the case showed that in December, 1900, the man had been an in-patient of the hospital suffering from phagedæna of the penis grafted on a Hunterian chancre under a phimotic prepuce. He was much debilitated, neglected, and in a foul condition on admission. However, with a prompt opening up of the phagedænic area, the application of pure phenol after removal of the sloughs, and continuous immersion in boric acid hip baths he rapidly improved though he lost much of his glans penis. When discharged from the hospital he was warned to attend for a regular course of treatment but was not seen again till he was admitted for the glaucomatous attack.

It is difficult to say what was the cause of this violent glaucoma which after a period of 24 hours ended in stoneblindness. The patient was very definite in his statement that he had never had any eye trouble beyond attacks of the common Egyptian ophthalmia and no history of iritis or atropine instillation was to be had, and except for the severe secondary syphilis I know of no cause. Glaucoma has been known to occur in eyes affected with hæmorrhagic retinitis and this retinitis has been known to occur in secondary syphilis, but against this as a cause must be set the fact that the patient was not himself aware of any previous loss of vision. The rapidity of the destruction of sight and the fact that there was no remission, complicated with the pus in the conjunctiva, rendered it a hopeless case from the onset, while the severity of the symptoms may to an extent be probabiy attributed to the cachexia of want and disease.

There is another point to which this case draws attention. It appears that this man had been living on the charity of the other Italian workmen employed on the dam by Messrs. Aird as he was without money, having been out of work. Previously to his first admission in December he had been for some time out of work and owing to the nature of his malady had not been drawing sick pay from the sick fund jointly provided by Messrs. Aird and the men, which was by ts rules not available for persons suffering from venereal disease. Moreover, according to the strict rule of the hospital treatment for such cases was supposed to be paid for by the men and not provided gratis, and this was the reason the man gave for not attending for further treatment of the syphilis. The result of all these conditions was hat he got neither proper food nor treatment, and such a ase to my mind goes a long way to support the contention which has been brought forward so often at continental congresses, that it is really in the end false economy speaking in the wider sense of the relations of the individual to the general cosmos) to legislate either privately or publicly in a repressive and hostile sense as regards venereal disease. It is true that these diseases are contracted as the result of self-indulgence, but there is a great deal to be said for those who contend that it results in economic waste to put undue obstacles in the way of free treatment for these persons, who deserve attention not as criminals but as persons suffering from a disease which is not only highly contagious but in one of its forms may be transmitted to offspring. At present in England this view is not popular either with the profession or with those whose work brings them in contact with this question, but there is a good deal to be said for the proposition that when the Magdalene of the lock hospital and the blennorrhoic of the out-patient room are treated by pious surgeons and mission ladies less as criminals and more as sick persons a decided step will be taken on what many think the more sound and humane way.

Assiout.

\section{NOTE ON A CASE OF TETANUS; INJECTIONS OF ANTITETANIC SERUM ; RECOVERY.}

By Daniel Mowat, M.D. Edin.,

CHIET CLINICAL ASSISTANT, ROYAI LONDON OPHTHALMIC HOSPITAL.

A WOMAN, aged 40 years, the wife of a farrier, whilst walking across the floor of her husband's forge trod upon a block of wood from which a large nail protruded about one and three-quarter inches. She was wearing soft felt shoes and the nail penetrated into the ball of the left great toe about one inch. I saw her on Sept. 3rd, 1903, and, judging by the pain on moving the great toe, the nail had penetrated the metatarso-phalangeal joint. The leg was very painful and somewhat swollen; the wound was septic and inflamed, movement of the toe causing great pain. Antiseptic dressings were applied to the wound and a mixture of chloral hydrate, morphine, and bromide of potassium was given to relieve pain and to induce sleep.

On Sept. 4th the patient complained of a feeling of stiffness in the muscles of the jaws and pain up the back. The pain in the toe was still severe but she had slept a few bours after taking the medicine. During the next few days the wound seemed to be clean and there was little pain on movement. The feeling of stiffness in the jaws had nearly passed away. On the 9 th and 10th the patient sat up for some hours, saw a few friends, and did household work contrary to orders, as I somewhat feared tetanus setting in. In spite of the medicine she did not sleep on the 10th and on the following day there was well-marked rigidity of the masseters and also of the muscles of the back, the abdomen, and the thighs. The jaws could scarcely be separated. On the following day the spasms were well marked and opisthotonos was present. The pain was very great and the patient was unable to speak. Antitetanic serum was procured from Messrs. Allen and Hanbury and ten cubic centimetres were injected into the abdominal walls. The patient had a quieter night but the spasms were very frequent. On the 13th another ten cubic centimetres of serum were injected, after which the spasms became less frequent. A red rash developed for some distance over the abdominal walls where the injections had taken place. On the 14th the patient was quieter but begged for the injection of serum as she experienced great relief after half an hour. As she was much better on the 15th no injection was given. On the 16th she was not so well, the jaws were firmly clenched, and the abdominal muscles were very rigid. Another ten cubic centimetres of antitetanic serum were injected. On the $17 \mathrm{th}$ and $18 \mathrm{th}$ the various muscles were still rigid but less painful. She was fed during the last few days through the trube of an ordinary feeding bottle passed 